\title{
IDENTIFICACIÓN DE COMPETENCIAS PROFESIONALES ACORDE CON LA PERSPECTIVA SOCIOFORMATIVA*
}

\author{
ANGELA TERESA BELTRÁN BUSTOS**, ANA MILENA ÁLVAREZ CANO*** \& \\ FELIPE HUMBERTO FERRO RODRÍGUEZ ${ }^{* * * * *}$ \\ UNIVERSIDAD AUTÓNOMA DE OCCIDENTE
}

Recibido/ Received/ Recebido: 17-08-2011 - Aceptado/ Accepted / Aprovado: 16-12-2011

\begin{abstract}
Resumen
Este artículo define las competencias para un programa académico, considerando los lineamientos curriculares y el posicionamiento estratégico formulado por la institución investigada. La metodología utilizada es la propuesta por Tobón (2010), la cual presenta 5 fases como ejes esenciales, en donde se hace una selección de competencias a partir de percepciones de diferentes grupos, como lo son: estudiantes, docentes, empresarios y egresados. Lo que condujo a una propuesta formativa con base en las nuevas competencias definidas que pretenden formar profesionales integrales, con un claro proyecto de vida, espíritu creativo, investigativo y capacidad de formular y desarrollar estrategias dentro de un entorno competitivo y retador. Se analizan de forma comparativa los currículos, lo que permite constatar los elementos diferenciadores que cada uno posee respecto de los demás. Como resultado se obtiene un panorama concreto sobre las competencias específicas del profesional investigado, lo cual es una sólida plataforma para la construcción colectiva de una propuesta curricular.

Palabras clave: Competencias genéricas, Competencias específicas, Currículo universitario.
\end{abstract}

\section{MARKETING AND INTERNATIONAL BUSINNES PROFESSIONAL SKILLS: SOCIAL AND TRAINING PERSPECTIVE}

\begin{abstract}
This article defines skills for an academic program, considering curricular guidelines and strategic positioning formulated by the research institution. The methodology used is the one proposed by Sergio Tobón, which presents 5 phases as essential axis, where there is a skill selection from perceptions of different groups, such as: students, teachers, entrepreneurs and graduated. This conducted to a formative proposal based on new defined competences which seek to form integral professionals, with a defined life project, creative and research spirit and capacity to formulate and develop strategies within a competitive and challenging environment. Curriculums are analyzed comparatively which allows verifying differentiating elements in relation to others. As a result, there is a concrete picture on specific competences of the professional, which is a solid platform for collective construction of a curricular proposal. Keywords: Generic skills, Specific skills, University curriculum, Marketing.
\end{abstract}

* Documento resultado de investigación realizada con el Programa Mercadeo y Negocios Internacionales de la Facultad de Ciencias Económicas y Administrativas de Universidad Autónoma de Occidente (Calí-Colombia) dentro del Diplomado en Pedagogía y Didáctica universitaria dictado por el Centro de Investigación en Formación y Evaluación CIFE. 2009-2010.

** Magíster en Administración de Empresas, Docente Universidad Autónoma de Occidente. Correo electrónico: atbeltran@uao.edu.co.

**** Magíster en Administración de Empresas con enfasis en mercadeo, Docente Universidad Autónoma de Occidente. Correo electrónico: amalvarez@uao.edu.co.

***** Magíster en direccionamiento estrategico, Director programa Mercadeo y Negocios Internacionales, Universidad Autónoma de Occidente. Correo electrónico: fhferro@uao.edu.co. 


\title{
QUALITICAÇÃO PROFESSIONAL EM MARKETING E NEGÓCIOS INTERNACIONAIS: PERSPECTIVA SOCIAL E DE FORMAÇÃO
}

\author{
Resumo
}

\begin{abstract}
Este artigo define as competências para um programa acadêmico, considerando as orientações curriculares e o posicionamento estratégico formulado pela instituição pesquisada. A metodologia utilizada é a proposta por Sergio Tobón, que apresenta 5 fases como eixos essenciais, onde se faz uma seleção de competências a partir de percepções de diferentes grupos, como estudantes, docentes, empresários e ex-alunos. Referido processo pode conduzir a uma proposta formativa baseada nas novas competências definidas que pretendem formar profissionais integrais, com um claro projeto de vida, espírito criativo, investigativo e capacidade de formular e desenvolver estratégias dentro de um meio competitivo e desafiador. Analisam-se de forma comparativa os currículos, o que permite constatar os elementos diferenciadores que cada um possui com relação aos outros. Como resultado se obtém um panorama concreto sobre as competências específicas do profissional pesquisado, o que é uma sólida plataforma para a construção coletiva de uma proposta curricular
\end{abstract}

Palavras chave: Competências genéricas, Competências específicas, Currículo universitário.

Beltrán, A.; Álvarez, A. \& Ferro, F. (2011). Identificación de competencias profesionales acordes con la perspectiva socioformativa. En: Revista de la Facultad de Ciencias Económicas de la Universidad Militar Nueva Granada. rev.fac.cienc.econ, XIX (2)

JEL: M30, M39.

\section{Introducción}

La formación profesional genera una reflexión permanente sobre las necesidades de los educandos, sobre la intervención orientadora, frente a cómo hacerla mas eficiente y cómo valorarla en el contexto del sector real. En el sistema educativo se hace imprescindible la formación de profesionales con orientación apropiada y la formación por competencias permite que los educandos adquieran comportamientos y destrezas en un ámbito especifico para desempeñarse de manera eficaz y satisfactoria, consistentes en la capacidad de vincular conocimientos teóricos con el saber hacer y el saber ser, facilitando el acercamiento al mercado laboral.

Por otra parte, el tema de los cambios en los proyectos de formación, se ha entendido como un pilar fundamental del proceso educativo, que busca la formación con óptima calidad. Es por eso, que las investigaciones en formación por competencias, son cada día mejores y más importantes produciendo cambios significativos en los planes de estudio.

Este trabajo precisa las competencias específicas para diseñar por competencias un currículo universitario en el área de Mercadeo y negocios internacionales. Se trata de un modelo centrado en el estudiante, que se basa en formar profesionales integrales y competentes, dado que la relación entre instituciones educativas y la sociedad se refiere a la posibilidad de asegurar que los conocimientos obtenidos en las aulas beneficiarán tanto el desarrollo de los estudiantes como el desarrollo de las organizaciones en el campo real. El artículo presenta los resultados del proceso de documentación y dialogo entre las partes interesadas, y como se concreta una propuesta sintética de competencias profesionales. El documento puede ser de referencia importante para el estudio e implementación de modelos de competencia en programas escolares cercanos. 


\section{El referente conceptual}

\subsection{El currículo}

Para el Ministerio de Educación Nacional (MEN) el currículo es un conjunto de criterios, planes de estudio, programas, metodologías y procesos que contribuyen a la formación integral y a la construcción de la identidad cultural nacional, regional y local, incluyendo también los recursos humanos, académicos y físicos para poner en práctica las políticas y materializaras en los Proyectos educativos institucionales PEI.

El Consejo Nacional de Acreditación (CNA) precisa la importancia de que el currículo sea lo suficientemente flexible para que, además de contribuir a la formación integral de los estudiantes, se adapte a las necesidades y vocaciones individuales y facilite una actualización permanente de contenidos y estrategias pedagógicas y la aproximación a nuevas orientaciones en los temas del programa.

Según Stenhouse (1988) se entiende por currículo "no un sumario o prescripción de objetivos, métodos y contenidos, es más bien un curso de acción, un objeto simbólico y significativo para maestros y alumnos que posee una existencia física, pero también un significado encarnado en palabras, imágenes, sonidos, juegos o lo que fuere; es un proceso educativo, una secuencia de procedimientos hipotéticos -bajo cierto enfoque epistemológico y ciertos criterios de enseñanza que sólo pueden comprenderse en una clase-. En este sentido el currículo es siempre hipotético".

Esto plantea una pregunta de la siguiente manera: ¿Qué debemos hacer para crear un currículo que posea esas cualidades de forma, armonía, principios, constancia y conformidad a estándares? (...) Y agrega: La enseñanza basada en la instrucción implica que la tarea a realizar consiste en que el profesor trasmita a sus alumnos conocimientos o destrezas que él domina. En la enseñanza basada en el descubrimiento, el profesor introduce a sus alumnos en situaciones seleccionadas o diseñadas de modo que presentan en forma implícita $\mathrm{u}$ oculta principios de conocimientos que desea enseñarles. Así, las regletas de Cuisenare corresponden a principios numéricos.
La instrucción y el descubrimiento resultan inadecuados en la clase siempre que se pueda especificar detalladamente el resultado que se desea obtener con la enseñanza y que dicho resultado sea aproximadamente el mismo para cada alumno (Stenhouse, 1991, 26, 69).

Por otro lado, Edgar Morin (1994) basa su propuesta en el interés en desarrollar un método complejo de pensar la experiencia humana recuperando el conocimiento descubriéndonos nosotros mismos, nuestras limitantes y nuestras posibilidades. El pensamiento argumenta, entre otros, que la Complejidad puede civilizar el conocimiento. Con su aplicación se puede llegar al desarrollo de la naturaleza humana multidimensional, la lógica generativa y la dialéctica conduciendo a un modo de construcción que aborda el conocimiento como un proceso biológico, cerebral, espiritual, lógico, lingüístico, cultural, social e histórico. Se dice que la realidad se comprende y se explica desde todas las perspectivas posibles. Se concibe que un fenómeno específico pueda ser analizado por medio de las más diversas áreas del conocimiento. Tanto la realidad como el pensamiento y el conocimiento son complejos y debido a esto, es preciso usar la complejidad para entender el mundo.

Hay tres principios sobre los cuales construye Morin (1996) lo que podría ser el paradigma de la complejidad: el principio de recursividad organizacional, el principio dialógico y el principio hologramático. La recursividad organizacional: Lo recursivo se refiere a proceso en los cuales los productos y los efectos son necesarios para su propia producción. El producto es al mismo tiempo el productor. Los problemas de organización social solo pueden comprenderse a partir de complejo en donde interactúan sociedades animales, células con su autonomía (particularmente para mercadeo este es un tema muy importante ya que la humanidad trae un prehistórico de recolector; impacto que afecta la acción del comportamiento del consumidor) produciendo un todo.

Por otra parte las interacciones entre individuos construyen a la sociedad. El principio dialógica: se basa en la asociación complementaria y antagonista de elementos imprescindibles para el funcionamiento de la organización y actuando a todos los niveles 
con una mediación entre orden y desorden, individuo y ámbito social, entre saber y barbarie. Principio hologramático: Este principio ve las partes en el todo y el todo en las partes, superar el principio de holismo (que solo ve el todo) y del reduccionismo (solo ve las partes).

A pesar de estas dificultades presentadas por Stehnhouse y Morin el Modelo "Developping a Currulum" DACUM afirma que el curriculum se debe orientar según el entorno por medio de un análisis ocupacional realizado por el participante. el cual analice ocupaciones profesionales por medio de la investigación con los integrantes directamente interesados. Afirma que existen poderosas razones para explicar el interés por implementar un enfoque orientado a las competencias en la formación de profesionales universitarios.

También es importante el Modelo Curricular de Schiro (1978), el cual identifica dos dimensiones bipolares, en el eje de "Y" positivo, se encuentra la atención a las fuentes del conocimiento y en el lado negativo se encuentra "la atención prevalente al uso del conocimiento". En el eje positivo se encuentra el "conocimiento puro de cada disciplina y la función practica en el desarrollo del alumno" y en el lado negativo; el "afecto y la prevalencia que adquiere la realidad objetiva frente a la subjetiva". Con base en esta teoría aparecen cuatro modelos distintos de escuelas curriculares: El centrado en las disciplinas y el aprendizaje formal o modelo academicista. El centrado en los intereses de los estudiantes, modelo humanista. El centrado en el cambio social, modelo crítico y el centrado en la eficacia, modelo tecnológico.

\subsection{Concepto de competencia en la educación}

Este concepto hace referencia a una formación integral del profesional como ciudadano por medio del aprendizaje significativo en el saber, el saber - hacer $y$ el saber ser en un contexto, en donde se requiere del conocimiento teórico y se convierte en un compromiso de cooperación en la práctica expresado en el desempeño. Sin embargo, la competencia no se reduce al desempeño laboral sino que abarca a un ser responsable que soluciona problemas con diferentes niveles de complejidad.
Le Bofert (1994) describe las competencias como la capacidad de movilizar y aplicar correctamente en un entorno laboral determinado, recursos propios como habilidades, conocimientos y actitudes y recursos del entorno para producir un resultado definido.

Para Rey (1996) la competencia es la "capacidad de generar aplicaciones o soluciones adaptadas a cada situación, movilizando los propios recursos y regulando el proceso hasta lograr la meta pretendida. Este autor distingue las competencias como conductas: "capacidad para cumplir una tarea determinada"; y la capacidad como función: "sistema de conocimientos conceptuales y procedimentales organizados como esquemas operacionales que permiten, frente a una familia de situaciones, la identificación de un problema y su resolución mediante una acción eficaz".

Según Sladogna (2000) toda competencia es una síntesis de las experiencias que el sujeto ha logrado construir en su entorno trascendental en el pasado y presente. Las competencias son capacidades complejas que poseen distintos grados de integración y se manifiestan en una gran variedad de situaciones en los diversos ámbitos de la vida humana personal y social. Así mismo, Echeverria et al. (2001) describe la competencia como aquella que posee un profesional quien dispone de los conocimientos, destrezas y actitudes necesarias para ejercer actividad laboral, y está capacitado para colaborar en su entorno y en la organización del trabajo.

Otra visión es la de la OECED (2003) que plantea que la capacidad para responder exitosamente una demanda compleja o llevar a cabo una actividad o tarea, incluyendo las actitudes, valores, conocimientos y destrezas que hacen posible la acción efectiva es una competencia.

En el año de 2004 la Comisión Europea definió a las competencias como aquellas que "Se refieren a una combinación de destrezas, conocimientos, aptitudes y actitudes, para aprender, además del saber. También incluyen como parte importante la disposición para adquirir las competencias y agrega que estas deberían haber sido desarrolladas para el final de la enseñanza o formación obligatoria, y deberían 
actuar como la base para un posterior aprendizaje como parte de un aprendizaje a lo largo de la vida." Comisión Europea (2004).

El término competencia ha tenido diversas interpretaciones y se ha utilizado para referirse a múltiples situaciones. La educación sugiere que el aprendizaje puede ser más eficaz cuando ocurre en un contexto donde el conocimiento es ubicado fundamentalmente como un resultado de la actividad donde este se desarrolla. En este marco las Competencias genéricas se refieren a competencias transversales y transferibles a multitud de áreas y profesiones se incluyen elementos de orden cognitivo, actuacional y motivacional, y se expresan a través de las denominadas competencias instrumentales, de orden metodológico o de procedimiento; competencias personales, tales como el trabajo en equipo, el manejo de las relaciones interpersonales, el compromiso ético; y las competencias sistémicas, que se manifiestan en el aprendizaje autónomo.
Las Competencias específicas, son competencias relacionadas directamente con una ocupación o profesión. En este sentido, (Corominas, 2001, 307) expresa:

"La preparación profesional abarca, tanto la formación o entrenamiento en competencias específicas de la profesión, es decir, saberes y técnicas propias de un ámbito profesional (por ejemplo: interpretar un gráfico de temperaturas y lluvias, calcular la resistencia de un forjado, evaluar los conocimientos adquiridos por un alumno, gestionar créditos a clientes, etc.), como el entrenamiento en competencias genéricas comunes a muchas profesiones (por ejemplo: gestión de la información, adaptación a los cambios, disposición hacia la calidad, etc.). Las competencias específicas están más centradas en el «saber profesional», el «saber hacer»y el «saber guiar» el hacer de otras personas; mientras que las competencias genéricas se

Tabla 1: Algunos modelos de currículos por competencias ${ }^{1}$

\begin{tabular}{|c|c|c|}
\hline Fuente & Modelo & Breve explicación \\
\hline $\begin{array}{l}\text { González \& Wagenaar } \\
\text { (2003) }\end{array}$ & $\begin{array}{l}\text { proyecto Alfa } \\
\text { TUNING }\end{array}$ & $\begin{array}{l}\text { Identifica las competencias genéricas precisando que cada titulación busca cubrir competencias más especí- } \\
\text { ficas, es decir más pertinentes a cada área temática. Tuning para America Latina propone doce áreas temáticas } \\
\text { y llega a un consenso sobre las competencias relativas de cada área. }\end{array}$ \\
\hline $\begin{array}{l}\text { Programa de formación para } \\
\text { la nivelación académica } \\
\text { de maestros en servicios } \\
(2003)\end{array}$ & $\begin{array}{l}\text { el Modelo } \\
\text { de DeSeCo, } \\
\text { (OCDE) }\end{array}$ & $\begin{array}{l}\text { Propone identificar competencias instrumentales, interpersonales y sistémicas siendo más participativo y } \\
\text { utilizando instrumentos de forma interactiva, en grupos heterogéneos de forma autónoma. }\end{array}$ \\
\hline $\begin{array}{l}\text { Chyung, Stepich \& Cox } \\
\text { (2006). }\end{array}$ & $\begin{array}{l}\text { Instructional } \\
\text { Perfomance } \\
\text { Technology de } \\
\text { la Boise State } \\
\text { University }\end{array}$ & $\begin{array}{l}\text { Desarrolla su currículo basado en competencias según la realidad empresarial. Este proceso, se inicia con el } \\
\text { análisis de la industria y los egresados, se investigan los modelos profesionales por medio de benchmarking } \\
\text { y se revisa el currículo para después diseñar los módulos de estudio y evaluar su efectividad. } \\
\text { Si bien el modelo de la Instructional Perfomance Technology de la Boise State University desarrolla su currí- } \\
\text { culo basado en competencias según la realidad empresarial el vacío esta en que no incluye las opiniones de } \\
\text { los docentes que conducen a la verificación del conocimiento y dominio de saberes. Esta retroalimentación } \\
\text { es importante porque los docentes son quienes hacen la evaluación progresiva del aprendizaje permitiéndoles } \\
\text { identificar las dificultades en el proceso de aprendizaje y detectar deficiencias. }\end{array}$ \\
\hline $\begin{array}{l}\text { Universidad Católica de Co- } \\
\text { Iombia (2005). }\end{array}$ & Modelo UCAC & $\begin{array}{l}\text { Se enfoca en el desarrollo de las competencias generales y competencias profesionales propias de la carrera. } \\
\text { La estructura del modelo comprende el enunciado y definición de la competencia, el enunciado y definición de } \\
\text { las unidades, el criterio de desempeño y los indicadores que permitan evaluar el dominio de la competencia. } \\
\text { Las competencias generales se basan en el modelo Tuning y compilan } 4 \text { dimensiones, aprender a aprender } \\
\text { con calidad; aprender a trabajar con el otro; aprender a interactuar en el contexto global y aprender a convivir } \\
\text { y a servir. }\end{array}$ \\
\hline
\end{tabular}

1 Fuente: Elaboración propia. 
sitúan en el «saber estar»y el «saber ser». Son transferibles en el sentido de que sirven en diferentes ámbitos profesionales".

Finalmente, Tobón (2010) propone entender las competencias como actuaciones integrales para identificar, interpretar, argumentar y resolver problemas del contexto con idoneidad, ética y mejoramiento continuo.

\section{Modelos de currículos por competencias}

Las principales fuentes que originan los modelos curriculares son la sociedad, la cultura, las organizaciones y la academia, en los que se diferencian $e$ integran las competencias genéricas y específicas de las áreas disciplinares. Algunos de los modelos curriculares cimentados en competencias son presentados en la tabla No. 1.

Otros referentes son el modelo Europeo en 2005, en el que Bélgica va más allá que del Modelo DeSeCo porque incluye la imagen positiva de si misma y el ser capaz de actuar y pensar de manera independiente así como la agilidad mental. El modelo curricular por competencias de Alemania e Irlanda, el cual está centrado en aprender a aprender uniendo los conocimientos temáticos, se diferencian en que el de Irlanda incluye el trabajo en equipo y la eficacia personal y Alemania no lo incluye.

Sin embargo, en los modelos analizados no se observa un método que incluya todos los entornos involucrados en el desempeño eficiente del profesional, tampoco presentan un modelo formal que permita configurar las competencias y realizar un proceso de selección de las mismas, de allí que este trabajo se centre en el modelo socio formativo.

El modelo que se propone en este documento es capaz de sustentar un procedimiento de información que registra los datos necesarios para la identificación de las competencias especificas, involucrando todas las partes interesadas, con miras a efectuar modificaciones el la malla curricular. La socio formación es desarrollarse de manera integral, con un proyecto ético de vida sólido, en el marco de las relaciones colaborativas con otras personas, afrontando los retos actuales y futuros del contexto y contribuyendo al tejido social, al desarrollo organizacional, a la economía, a la cultura y al equilibrio ecológico ambiental. La base es el pensamiento complejo de Morin el cual consiste en entretejer las cosas entre si en el ámbito de relaciones de organización con flexibilidad y creatividad. Desde la socio-formación las competencias son procesos integrales de actuación ante actividades y problemas de la vida personal y el contexto laboral profesional. También es la condición de compromiso de formación integral con un proyecto de vida sólido, entre personal contribuyendo al tejido social, al desarrollo organizacional a la cultura y al equilibrio ecológico ambiental. La base es el pensamiento complejo de Morin el cual consiste en entre tejer cosas entre si en el ámbito de las relaciones de la organización y el currículo de manera flexible y creativa hasta que se retroalimente.

\section{Metodología de la propuesta: el modelo socio-formativo}

Para la elaboración de competencias con el modelo socio-formativo se tomo como referente la propuesta de Tobón (2010) en donde el currículo es un proceso inteligente en el cual se han puesto de acuerdo las instituciones educativas y la sociedad frente a sus necesidades con respecto a la formación de competencias en función de los contenidos curriculares de las diferentes profesiones, cuyo interés es el de beneficiar la realización del estudiante, la construcción de la comunidad y el desarrollo económico. Tobón precisa que el diseño del currículo es un proceso investigativo de construcción conceptual y de aplicación, cuyo éxito en la calidad del plan formativo depende de la manera como se lleve a cabo. También aclara que para diseñar el currículo se requiere de una continua problematización e interrogación frente a su pertinencia contextual, pedagógica y filosófica; por lo tanto, el currículo siempre está en movimiento y se construye en el camino.

La propuesta metodológica utilizada es la de Tobón (2010) que se presenta en 5 fases, la autorreflexión, la deconstrucción, la investigación del concepto, el 
afrontamiento de la incertidumbre, y la identificación de las competencias, como ejes esenciales en la construcción del currículo por competencias.

\subsection{Fase A: Autorreflexión}

Se considera como punto de partida y trata de hacer un autoexamen de manera autocrítica. La academia en su quehacer pedagógico y bajo la condición de humano que la caracteriza, se va enfrentando a situaciones de aprendizaje que la obligan a tener posturas actualizadas que evolucionen los programas en forma positiva. Lo anterior conlleva a reflexionar, a realizar una autocrítica de manera organizada, permitiéndose abrir nuevos caminos para que sean utilizados frente a las diferentes situaciones que demanda la organización.

El currículo es una consecuencia del proceso de investigación cooperativo y la construcción de saberes. Desde el comité curricular se realizó un análisis del currículo actual en donde se motivó a la reflexión, al análisis y a la autocrítica como proceso de perfeccionamiento para la creación de la malla curricular que impacte en la formación de los profesionales de calidad.

\subsection{Fase B: De-construcción}

Una vez realizado una autocrítica acerca del currículo actual, corresponde secuenciar el análisis crítico del currículo del programa, el cual busca fortalezas y debilidades. Tomando como referencia Morín (1990) se realiza un análisis calificador del currículo del programa; este análisis permite identificar los puntos fuertes y los que están por mejorar.

También se elige el objeto de estudio y se mantiene su meta de mejorar o de cambiar algo, los participantes se comprometen con todos para que al término de la investigación, se compartan los resultados para fortalecer la calidad del currículo y establecer sus fortalezas y debilidades.

El análisis debe ser objetivo y en equipo en búsqueda de causales de las debilidades, de significados de las fortalezas y se pueden recolectar evidencias favoreciendo la reflexión, recomendando siempre mejoras o cambios.

\subsection{Fase C: Investigación del contexto}

Teniendo a la vista una primera aproximación a las causas de las debilidades y la identificación de las fortalezas se procede a identificar las necesidades actuales de formación, a partir del análisis del entorno, en el contexto externo y en el contexto interno. Para evaluar el contexto externo se realiza el análisis de los currículos y modelos pedagógicos de otras instituciones, normativas y orientaciones gubernamentales, así como las tendencias mundiales sobre modelos de educación, tecnológicas, ambientales y estudios cuantitativos de gremios y asociaciones.

Para evaluar el concepto de los empresarios y determinar que se debe reforzar en los planes de estudio se realiza unas estratificaciones homogéneas en empresas, grandes, medianas y pequeñas que hayan trabajado en proyectos específicos de la carrera, seleccionadas de los directorios empresariales de la Institución.

Para evaluar el contexto interno se revisan los aspectos institucionales, las causas de deserción o cancelación de asignaturas por parte de los estudiantes. También se realizan estudios exploratorios y descriptivos a egresados, empresarios, estudiantes, docentes $y$ directivos.

La información obtenida en la fase de recolección de datos permite realizar el análisis en dos etapas: primero se agrupan las competencias según las similitudes literales luego se tabulan según la frecuencia de cada respuesta, y finalmente se organizaron de mayor a menor puntaje. Recolección de datos, es un estudio de campo con:

- Opinión de los estudiantes. Previo a la opinión de los estudiantes y con base en el contexto externo, los directivos seleccionan una propuesta de al menos 20 competencias consideradas importantes, para el profesional del programa. Acto seguido se someten a votación para seleccionar las relevantes.

- Opinión de docentes, de egresados y de empresarios por medio de estudios exploratorios y descriptivos. 


\subsection{Fase D: Afrontamiento de la incertidumbre}

Una vez conocido el contexto interno y externo se enmarcó la necesidad de relacionar de una manera más estratégica, las necesidades de las organizaciones con el resultado académico, lo cual conduce a promover la implementación de las opciones basadas en los denominados modelos por competencias. Se presenta entonces el procedimiento que permite construir apropiadamente una competencia.

\subsection{Fase E: Identificación de las competencias}

Una vez se han realizado las etapas anteriores en donde se tienen, entre otros, las opiniones de los empresarios, los estudiantes, los egresados, los docentes y los directivos del programa se realiza un análisis global que permita conocer un panorama concreto sobre las competencias específicas del programa investigado. La identificación de competencias es la clave de la gestión investigativa y aporta una plataforma sólida para efectuar cambios en la malla curricular, generando el currículo basado en competencias.

\section{Construcción de competencias: caso currículo universitario en Mercadeo y Negocios internacionales}

Para una mejor compresión del proceso, en esta fase se aplican los pasos para la construcción de competencias para la elaboración del currículo por medio de un profundo trabajo desarrollado en un programa de Mercadeo y Negocios Internacionales.

\subsection{Autorreflexión}

En el programa se inició un proceso de autorreflexión sobre el currículo como consecuencia del proceso de investigación cooperativo y la construcción de saberes.

Las reflexiones de la educación como un factor fundamental del desarrollo del estudiante se extienden hasta impactar el desarrollo del comercio regional, nacional e internacional. A través de la actualización del currículo, el programa pretende ampliar su base cultural, satisfacer las necesidades de formación y perfilar profesionales actualizados de alta calidad pertinentes con las exigencias sociales. La posición del programa en el contexto internacional y en un marco de globalización creciente depende, en gran proporción, de su capacidad para propiciar la mejor formación posible de las personas que se proyectan una vez que pasan a ser egresados. El currículo por competencias es en la actualidad un eje importante en la transformación educativa con equidad y la llave para que cada docente y estudiante puedan desenvolverse en sus roles y ser asertivos en la sociedad del conocimiento. Conscientes de este nuevo contexto, el programa toma medidas orientadas a establecer las fortalezas y debilidades del servicio educativo en todos sus niveles y a propiciar innovaciones y cambios que reorienten la acción y que permitan asumir los nuevos desafíos.

En la evaluación del Proyecto Educativo Institucional, (PEI) se concluyó que el programa está en línea con los procedimientos y filosofías institucionales. Que la institución tiene un PEI sólido y altamente reconocido por la región. Los criterios para la evaluación del aprendizaje también son altamente conocidos y aceptados por la comunidad en general. En los aspectos pedagógicos se destacan la calidad como valor fundamental tanto para los alumnos como para la institución.

Las áreas curriculares preferidas son las investigaciones de mercados, fundamentos de mercadeo y mercadeo internacional, entre otros. Las cualidades del buen docente son: que sea un experto en la materia, que explique claramente, que sea cumplido y de calidad. En la metodología para mejorar la estrategia pedagógica se trabajó con clase magistral, metodología de casos, utilización de laboratorios, salidas educativas nacional e internacionalmente.

En los aspectos administrativos con relación a los recursos físicos, se prefirió el mejor acceso a las instalaciones, a los deportes y a las cafeterías. El área de bienestar universitario es valorado y tiene aceptación generalizada sin embargo aun desconocen todos los servicios de esta área. Desde el comité curricular se realizó un análisis del currículo actual en donde se motivó a la reflexión, al análisis y a la autocrítica en los entornos interno y externo, como proceso de perfeccionamiento para la creación de una malla 
curricular que impacte aún más, en la formación del profesional de calidad.

Lo anterior conllevó a reflexionar, a realizar una autocrítica de manera organizada, permitiéndose abrir nuevos caminos para que fueran utilizados frente a las diferentes situaciones que demandan las organizaciones.

Esta etapa se realizó con docentes de planta dedicados a investigar la práctica educativa del programa, también se analizaron datos y la problematización en si, algunas de las preguntas planteadas, fueron:

- ¿Cómo se manifiesta el currículo en las acciones de la práctica del egresado?

- ¿Cómo se relaciona el nivel del estudiante en la empresa con el aprendizaje universitario?

- ¿Cómo el egresado es capaz de seleccionar problemáticas y solucionar problemas?

Esta etapa es tarea difícil porque implica lo racional, lo emotivo del conocimiento en el pensamiento del profesor, sin embargo durante el desarrollo de la investigación se concentraron resultados que consolidan una propuesta de análisis de las competencias del estudiante, como mediación para facilitar la autorreflexión de la problemática.

\subsection{Deconstrucción}

Este fue un enorme proceso de reflexión como de construcción cultural al interior del programa, deliberando sobre sus pasos y decisiones sobre sus valores e importancia al identificar las principales competencias para la construcción del currículo. En el acto esencial de elegir y articular qué se transmitirá a cada nueva generación, en donde está en juego el futuro que se quiere construir. Concientes de que el currículum está en el núcleo de las relaciones para construir el cambio; tensado entre las variables internas y el entorno tanto nacional como internacional.

Se inició el proceso con miras a producir la propia malla curricular con importantes diferenciadores que determinan la primera fase de una larga secuencia de pasos con amplia participación de actores. Se analizaron entonces las fortalezas y debilidades del programa frente a:
- Misión y visión de la universidad

- Proyecto institucional

- Proyecto educativo del programa

- Relevancia académica y pertinencia social del programa

- Metodología pedagógica

- Interdisciplinaridad del currículo

- Calidad y desarrollo del docente

- Sistema de evaluación de estudiantes

- Investigación formativa

- Nivel de formación de profesores actuales

- Reingresos

- Numero y calidad de estudiantes

- Permanencia y deserción estudiantil

- Participación en actividades de formación integral

- Reglamento estudiantil

- Interacción nacional e Internacional

- Flexibilidad del currículo

- Proyección social

- Recursos Bibliográficos.

\subsection{Investigación del contexto}

\subsubsection{Contexto externo}

Se analizaron las siguientes tendencias:

- Tendencias mundiales de la educación: Para realizar este estudio y con el fin de observar y comparar las tendencias mundiales en relación con el perfil de lo que las empresas están demandando en un profesional de Mercadeo y Negocios internacionales, se seleccionaron y analizaron universidades de Latinoamérica, Europa y Estados Unidos.

- Tendencias económicas, sociales, tecnológicas, empresariales y educativas: Los componentes mundiales que para este milenio han venido trasformando la demanda laboral de profesionales universitarios, están conformados, en orden de importancia, por: la globalización, la transformación de las organizaciones, los avances tecnológicos y los nuevos aprendizajes, así:

I) La globalización: Esta nueva forma de moverse el mundo comenzó a gestarse en las tres últimas décadas del siglo XX, como una tendencia 
consistente e inevitable que ha logrado integrar económica, cultural y laboralmente a las naciones. Aspectos como los grandes avances en la sociedad de la información, donde se masifican la telefonía celular, los computadores personales y la Internet; el alcance, la cobertura y la calidad de las telecomunicaciones y el fortalecimiento de las conexiones económicas entre sectores y países reflejado en la multiplicación de los tratados de libre comercio, la liberación de los mercados de capitales y la creación y fortalecimiento de bloques regionales y transcontinentales, han originado nuevas formas de trabajo, exigiendo a las instituciones educativas cambios estructurales en el proceso de formación de los educandos.

II) La transformación de la función de las organizaciones: La globalización trajo consigo el fortalecimiento de las organizaciones transnacionales, tanto en los sectores productivos, como financieros y de servicios, convirtiéndose en las mayores poseedoras de patentes y tecnologías. En el contexto nacional, además de la llegada de multinacionales, se ha incrementado la participación de pequeñas y medianas empresas, el trabajo independiente y la prestación de servicios personales, entre otros.

III) Los avances tecnológicos: Estos han permitido que las compañías desarrollen nuevas formas de planear, organizar, dirigir y controlar todas las funciones de la empresa y, en especial, la comercialización de sus productos y servicios, los cuales se pueden producir, promocionar, publicitar y distribuir a partir de redes virtuales.

IV) Los nuevos aprendizajes: La población joven, influenciada por un entorno globalizado más acelerado, más exigente y más competitivo, ha desarrollado otras formas de aprendizaje. Según un estudio sobre medios digitales y aprendizaje realizado en los Estados Unidos y financiado por la Fundación MacArthur (2008) ${ }^{2}$, las actividades importantes para el desarrollo de los adolescentes son: Navegar por Internet, jugar en la computadora y pasar tiempo interactuando en redes sociales, siendo estas las herramientas que están empleando para relacionarse y para estudiar; es decir, que el aprendizaje hoy en día depende más de los compañeros y de las redes sociales, y esto es algo importante que debemos considerar si queremos repensar la educación del siglo XXI.

Lo anterior permite concluir que las organizaciones se están transformando y preparando para responder más rápida y efectivamente a estos nuevos retos, lo que significa que serán superiores a estas exigencias aquellas organizaciones que reconozcan la comunicación, la innovación y la tecnología, las relaciones internacionales, el mercadeo y la cualificación del talento humano como variables obligatorias en su planeamiento estratégico.

En este contexto, el sector educativo, y en especial las instituciones de educación superior, juegan un papel protagónico, puesto que tienen la responsabilidad social de entregar a las organizaciones, al Estado y a la comunidad personas integrales y altamente competitivas, que propendan a su desarrollo económico, social y cultural, y que en realidad contribuyan, en orden de importancia, con el incremento del nivel competitivo de nuestros países. Esto quiere decir que las instituciones deben presentar ofertas de formación acordes con las necesidades que demandan los diferentes actores que conforman el sistema social.

\subsubsection{Oferta educativa de programas de Mercadeo y afines en Colombia}

Para la presentación de la información y el análisis de la oferta de educación superior en Colombia, el Ministerio de Educación Nacional agrupa las carreras o programas académicos de acuerdo con su afinidad temática o con la cercanía de sus contenidos, con los campos específicos del conocimiento y con los campos de acción, ocupación y desempeño profesional a los que conducen. Estos campos se denominan Núcleos Básicos de Conocimiento (NBC) los cuales ascienden a 55 NBC y se agrupan en ocho Áreas de Conocimiento. En la Ilustración 1 se muestra que el mayor número de títulos otorgados se concentra en el área de Economía-Administración-Contaduría y afines (Mercadeo) con un 31,3\%, equivalente a 425.895; seguida del área de Ingeniería-Arquitec-

2 Tomado de: www.macfound.org/ 
tura-Urbanismo con un $23,7 \%$, igual a 323.303 ; y de Ciencias Sociales y Humanas, con un $18,5 \%$, correspondiente a 252.116. Las áreas de Matemáticas y Ciencias Naturales y de Agronomía-Veterinaria y afines participan con el 1,6\% (equivalente a 21.698) y $1,4 \%$ (igual a 19.548), respectivamente.

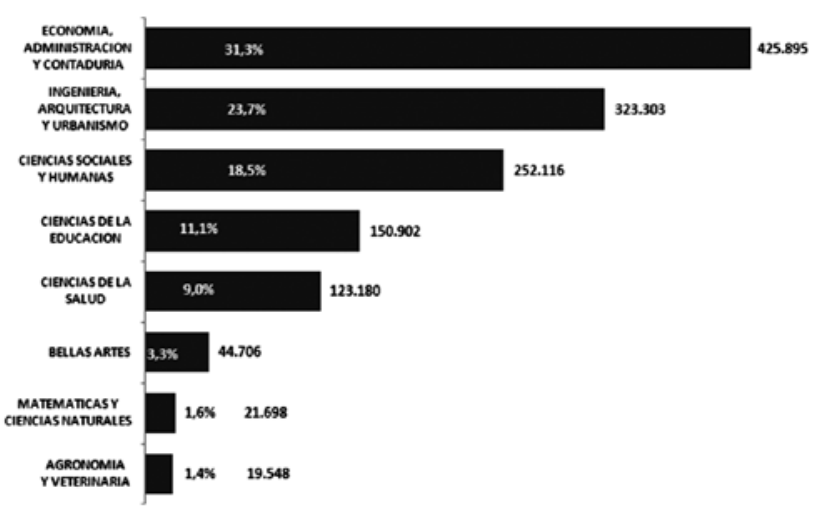

Ilustración 1. Graduados de educación superior por áreas de conocimiento (2001- 2009)3

En relación con los programas de Mercadeo y afines, el Ministerio de Educación Nacional de Colombia, en su página del Sistema de Prevención y Análisis de la Deserción en las Instituciones de Educación Superior (SPADIES), registra 99 programas, tanto en formación tecnológica como profesional, pertenecientes a instituciones de educación superior y universidades.

Tabla 2. Caracterización de la formación profesional en Colombia 4 .

\begin{tabular}{|l|c|}
\hline Programa Universidad acreditada & 1 \\
\hline Universidades con registro calificado & 19 \\
\hline Instituciones universitarias con registro calificado & 14 \\
\hline Instituciones universitarias sin registro calificado & 24 \\
\hline Tecnológicos acreditados & 2 \\
\hline Tecnológicos sin registro calificado & 15 \\
\hline Tecnológicos con registro calificado & 24 \\
\hline Total programas de Mercadeo & $\mathbf{9 9}$ \\
\hline
\end{tabular}

Las denominaciones de programas ofrecidos con más frecuencia por las universidades de Colombia son: Mercadeo, con un 74\%; seguida de Mercadeo y Publicidad, con $16 \%$; y el $10 \%$ restante corresponde a programas con denominación particular.

En las instituciones universitarias Colombianas, las denominaciones de los programas profesionales presentan gran diversidad, observándose una tendencia hacia el Mercadeo, de tal manera que 14 instituciones ofrecen solamente Mercadeo, tres Mercadeo y Publicidad, dos Mercadeo Internacional y una Mercadeo Agroindustrial. Es importante resaltar que se registran un gran número de programas en las instituciones universitarias que a la fecha no han logrado el registro calificado.

En conclusión, se observa que entre todos los programas de pregrado ofrecidos en Colombia predomina la denominación de Mercadeo; en segundo lugar está Mercadeo y Publicidad. Esta tendencia responde en gran parte a la necesidad manifestada por los empresarios en cuanto a que un profesional en mercadeo debe contar un amplio conocimiento de publicidad.

\subsubsection{Distribución de los programas de Mercadeo en Colombia}

En cuanto a la distribución geográfica de los programas de mercadeo en Colombia, Bogotá tiene el primer lugar, con un 37\%, explicable por el gran tamaño de su población; Medellín tiene la siguiente posición, con un porcentaje del $35 \%$, poco entendible por su volumen de población pero explicable porque esta es una región fuerte en mercadeo internacional. En Cali se ha presentado en los últimos 10 años un incremento en la oferta de programas de Mercadeo $y$ afines; a la fecha existen 4 programas profesionales ofrecidos por Universidades. Por medio de este análisis, el cual es de elaboración propia, se concluye que la carrera tiene alta aceptación en el país y que las principales universidades la ofrecen.

\footnotetext{
3 Fuente: Observatorio Laboral para la Educación. Graduados Colombia (2010). Recuperado de: < http://www.graduadoscolombia. edu.co/html/1732/article-195063.html>

4 Elaboración propia con información obtenida del Sistema Nacional de Información de la Educación Superior (SNIES).
} 


\subsubsection{Tendencias del perfil del Mercadólogo en el contexto Internacional y Nacional}

La crisis económica que vienen enfrentando países como Estados Unidos y algunos europeos como España y Grecia, ha obligado a sus dirigentes a cambiar las políticas y estrategias, tanto de organizaciones estatales como privadas. Es así que para las empresas ya no es prioridad el marketing de afuera hacia adentro sino que se analizan los costos, se proponen programas de reducción de gastos y se aplican modelos económicos para trabajar de forma rentable. En la actualidad se requieren departamentos de mercadeo que operen de manera integral con las demás áreas de la empresa.

Finalmente, los empresarios concluyen que el problema está en los planes de estudio; en ellos debe fortalecerse la base epistemológica y una formación en investigación, al menos para que los profesionales en Marketing puedan tener un conocimiento predictivo de la conducta del consumidor.

Ante estas críticas, las empresas, las universidades y los profesionales de mercadeo han venido reflexionando, para determinar que se deben reforzar los planes de estudios en cuatro aspectos que ya están siendo acogidos a nivel internacional:

I. Deben relacionarse con las áreas de finanzas, contabilidad y ventas, dado que la esencia de los negocios es la creación de valor para el cliente y para la empresa.

II. Deben incorporar las modernas tecnologías de la comunicación para abrirse a una mercadotecnia relacional. La estrategia es conquistar, conocer y retener a los clientes.

III. Tienen que profundizar en el conocimiento previo del comportamiento del consumidor; este conocimiento debe ir más allá del comportamiento: se debe investigar sobre contexto cultural, sobre la relación sociedad-consumo y sobre los valores.
IV. Deben considerar los procesos como un sistema que genere conciencia social y comprender que son responsables de los satisfactores de las necesidades desde el momento en que se concibe la idea, se transforma en bien, se entrega al consumidor, hasta la disposición final de los desperdicios o desechos generados, para recuperarlos o velar por que no perjudiquen ecológicamente al planeta o socialmente a las personas que lo habitan.

Por medio de un análisis que pretendió conocer la tendencia de las estructuras curriculares se realizó un estudio de los currículos de algunas universidades en Latinoamérica, dos de EE. UU. y dos de Europa, según la metodología propuesta. Se encontró que en la formación profesional predominan los cursos de introducción al mercadeo, mercadeo de los servicios, mercadeo estratégico, plan de mercadeo, mercadeo social, mercadeo en Internet, mercadeo ecológico, mercadeo internacional, psicología del consumidor, producto, promoción, precio e investigación de mercados. En el componente administrativo están la introducción a la administración, relaciones públicas, recursos humanos, gerencia empresarial, creación de empresas, derecho comercial y aspectos legales administrativos. La formación básica está basada principalmente en matemáticas, cálculo y estadística. En otras asignaturas se destacan la ética y la creatividad.

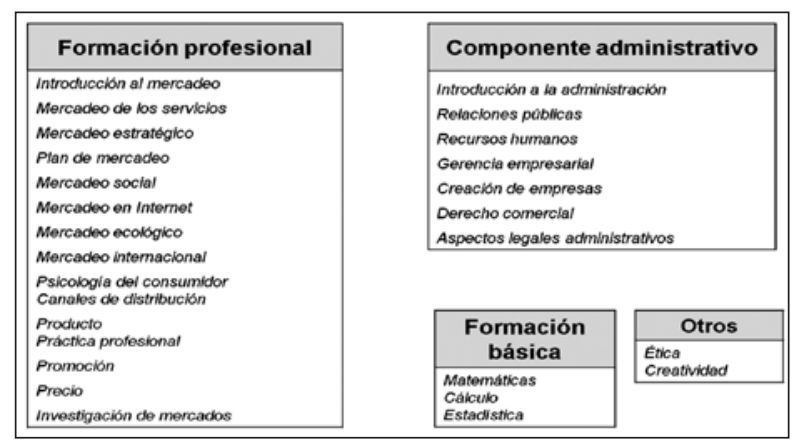

Ilustración 2. Análisis de estructuras curriculares nacionales e internacionales. Componentes dominantes ${ }^{5}$.

Como se puede observar en la Ilustración 2 la situación de las universidades en el mundo, ofertantes

5 Fuente: Elaboración propia. 
de los programas de Mercadeo presentan una gran claridad en la comprensión de los términos, así como en la forma de apropiarse de algunas materias de formación profesional, ya mencionadas. Con mayor frecuencia en las observaciones, y que tienen que ver directamente con la apropiación del componente administrativo curricular, se hace énfasis en 7 asignaturas. Otros componentes son la formación básica, como las matemáticas, cálculo y estadística considerados de política curricular de importancia en los procesos pedagógicos. Se pudo visualizar que en interior del campo del currículo se desarrollan pensamientos similares que presentan un conjunto de elementos compartidos, muchos de ellos derivados de lo que se puede reconocer como un trabajo académico, así como una focalización de los temas que se incluyen en el ámbito curricular. Sin embargo, cada universidad se encuentra en una lucha contra las demás del campo, en cuanto a estado de conocimiento y a través de la pertinencia institucional.

\subsection{Contexto Interno}

\subsubsection{Análisis. Estudio de campo}

La información obtenida en la fase de recolección de datos permitió realizar el análisis en dos etapas: primero se agruparon las competencias según las similitudes literales luego se tabularon según la frecuencia de cada respuesta, y finalmente se organizaron de mayor a menor puntaje. A continuación se presentan los resultados definitivos:

\section{- Opinión de los estudiantes}

Para los estudiantes, es muy importante que en su formación se fortalezcan competencias relacionadas con un conocimiento claro del mercadeo y las tendencias del consumidor y de los mercados, cómo realizar negociaciones internacionales, diseñar estrategias y productos con responsabilidad social, manejar técnicas cualitativas y cuantitativas de información, y las tecnologías de la comunicación.

Igualmente mencionan que todo profesional debe manejar un segundo idioma como el inglés; comunicarse efectivamente, tanto de forma oral como escrita; resolver problemas y trabajar en equipo.

\section{- Opinión de los docentes}

La opinión de los docentes coincidió con la opinión de los estudiantes en cuanto al conocimiento y utilización de las técnicas de investigación cualitativa y cuantitativa de información, como herramientas para conocer las necesidades del consumidor y las tendencias del mercado, así como el manejo de análisis cuantitativos de información como costos y finanzas.

Ante las preguntas:

¿Cómo se manifiesta el currículo en las acciones de la práctica del egresado? Los docentes opinaron que es definitivo, los empresarios esperan estudiantes que tengan claridad en las funciones pertinentes con su profesión y esperan apoyarse en ellos para agilizar su competitividad.

¿Cual es el tipo de relación entre el nivel del estudiante con la empresa frente a la empresa y el aprendizaje universitario? Opinaron que los estudiantes de semestres intermedio se relacionan muy tímidamente y le cuesta trabajo asumir el rol rápidamente; los estudiantes de últimos semestres se adaptan mejor a la practica universitaria.

¿Cuál es la capacidad de un egresado para seleccionar problemáticas y solucionar problemas? El docente cree que inicialmente el estudiante es una esponja que absorbe toda la información de la empresa y que en su etapa de práctica es un aprendiz. Pero también cree que rápidamente se involucra y empieza a identificar problemas y generar soluciones a estos problemas.

\section{- Opinión de los egresados}

Los egresados consideran que lo que debe poseer un profesional para ser competitivo en el mercado es la capacidad de análisis, habilidad para resolver problemas, manejar costos, presupuestos y finanzas, comunicarse en inglés, conocer técnicas avanzadas de investigación de mercados, conocer al consumidor y diseñar estrategias de publicidad y de marketing.

\section{- Opinión de los empresarios}

Para lograr la relación definitiva de las competencias más importantes para los empresarios se consideraron dos fuentes de información: Una, la entrevista 
realizada a gerentes y propietarios de empresas donde laboran egresados del programa y dos, estudio de los perfiles más requeridos por las empresas en Colombia y en Latinoamérica.

\subsubsection{Afrontamiento de la incertidumbre}

La experiencia pedagógica, unida a las diversas reflexiones de los diferentes escenarios, se siembra y fructifica con fuerza como parte estratégica en los trabajos de los diferentes actores del proceso, apoyados en herramientas que se construyen para poder crear las competencias de una manera estructurada y con criterios de fondo. La tabla 3 se elabora, a manera de ejemplo, con el fin de ayudar a comprender la naturaleza de una competencia y de su construcción.

\section{Identificación de las competencias}

\subsection{Definición de las competencias especificas del profesional}

El análisis global de las opiniones presentadas y de la investigación permitió conocer un panorama concre- to sobre las competencias específicas del profesional en Mercadeo y Negocios Internacionales, como son:

- Gestionar estrategias de mercadeo en contextos nacionales e internacionales, para elevar la competitividad de la organización, considerando modelos establecidos.

- Identificar las necesidades del consumidor y las oportunidades o los riesgos de mercados nacionales e internacionales, para el diseño e implementación de estrategias de desarrollo de marcas, distribución de productos y logística.

- Optimizar la utilización de los recursos asignados para el direccionamiento de las estrategias de mercadeo, considerando la interpretación, análisis de los costos y la información financiera.

- Utilizar las nuevas tecnologías de información y comunicación para conocer las necesidades de los clientes, desarrollar marcas y negocios, diseñar e implementar estrategias de comunicación y de comercialización a través de la red de acuerdo con el contexto.

Tabla 3. Descriptor de Competencia: Conocimientos fundamentales de Mercadeo ${ }^{6}$.

\begin{tabular}{|c|c|c|c|}
\hline $\begin{array}{l}\text { Verbos de desempeño } \\
=\text { actuación }\end{array}$ & $\begin{array}{l}\text { Objeto } \\
\text { (EI verbo actúa } \\
\text { sobre este objeto) }\end{array}$ & Finalidad & Condición de referencia \\
\hline $\begin{array}{l}\text { Interpreta, argumenta y resuelve } \\
\text { problemas. }\end{array}$ & $\begin{array}{l}\text { Conocimientos fundamenta- } \\
\text { les de Marketing }\end{array}$ & $\begin{array}{l}\text { Comprender, crear y em- } \\
\text { prender proyectos. }\end{array}$ & Textos de reseña en este campo de acción. \\
\hline \multicolumn{2}{|l|}{ Descripción de la competencia } & \multicolumn{2}{|c|}{$\begin{array}{l}\text { Aplica los conocimientos fundamentales del mercadeo para interpretar, argumentar, resolver } \\
\text { problemas y realizar proyectos con creatividad y responsabilidad social, acordes con los re- } \\
\text { ferentes de este campo. }\end{array}$} \\
\hline \multirow{5}{*}{ Criterios que apoyan esta compe } & \multirow{5}{*}{ encia } & \multicolumn{2}{|c|}{ Conceptualiza de acuerdo de acuerdo con los referentes teóricos del mercadeo. } \\
\hline & & \multicolumn{2}{|c|}{$\begin{array}{l}\text { Interpreta problemas del área de mercadeo de acuerdo con los conocimientos fundamentales } \\
\text { de esta área. }\end{array}$} \\
\hline & & \multicolumn{2}{|c|}{ Argumenta problemas en el área de mercadeo de acuerdo con los conceptos esenciales. } \\
\hline & & \multicolumn{2}{|c|}{$\begin{array}{l}\text { Plantea soluciones generales a problemas básicos en el mercad4eo de acuerdo con los con- } \\
\text { ceptos fundamentales del área. }\end{array}$} \\
\hline & & \multicolumn{2}{|c|}{ Responde con los planes establecidos en tiempo forma y lugar de manera efectiva. } \\
\hline
\end{tabular}

6 Fuente: Elaboración propia 
- Realizar negocios internacionales para generar sostenibilidad y desarrollo a las organizaciones, tomando como referencia los conocimientos sobre comercio y negocios internacionales.

\section{Resultados finales}

El presente documento define el método para encontrar las competencias de un programa académico profesional, su contenido es el resultado de procesos de consulta que involucraron: Docentes, empresarios, estudiantes, egresados, expertos y especialistas. Se organizaron foros, encuestas, entrevistas y discusión de los perfiles profesionales y las bases curriculares en las distintas áreas profesionales involucradas hasta el momento en un programa de Mercadeo.

Los resultados tienen una doble función:

- Mostrar un modelo susceptible de ser empleado en cualquier programa profesional universitario debido a la interrelación que mantienen entre si con las actividades académicas y sus actores. Los beneficios, que reporta el modelo al ser aplicado, forman una especie de vínculo entre actores que analizan y evalúan al profesional de cualquier profesión sobre, cuales serán las mejores competencias para su desempeño futuro.

- Definir competencias profesionales para un programa de Mercadeo que se tomarán como estándares para la construcción de un currículo con validez nacional e internacional. El modelo de Sergio Tobón establecido para mostrar las competencias ideales de un currículo y el método aplicado han sido válidos evidenciando como resultado las competencias profesionales.

Esta metodología permitió distinguir claramente la descripción y los criterios de cada competencia seleccionada por grupos líderes de opinión en el asunto. Las universidades estudiadas y los empresarios ayudaron de forma importante a documentar el panorama del currículo. La metodología propuesta ofrece una buena aproximación para la elaboración por competencias de un currículo universitario como lo muestran los resultados obtenidos en la selección de las cinco competencias descritas para el profesional en Mercadeo:

- Gestionar estrategias de mercadeo en contextos nacionales e internacionales.

- Identificar las necesidades del consumidor y las oportunidades o los riesgos de mercados nacionales e internacionales.

- Optimizar la utilización de los recursos asignados para el direccionamiento de las estrategias de mercadeo.

- Utilizar las nuevas tecnologías de información y comunicación.

- Realizar negocios internacionales para generar sostenibilidad y desarrollo a las organizaciones.

En cuanto a la amplia distribución geográfica de los programas de mercadeo en Colombia, son conclusiones que aportan en el desarrollo de las competencias del programa debido a que el interés nacional, acerca de la carrera esta relacionado con todas las carreras que tienen afinidad en el nombre y la malla académica. Este análisis es un resultado muy importante para la estructura curricular, porque se ve el interés de formar recurso humano calificado a la luz de procesos específicos relacionados con el Mercadeo, siendo ésta una estrategia de gran impacto en el desarrollo de la competitividad de las organizaciones nacionales, regionales e internacionales.

Como resultado del análisis de las estructuras curriculares en universidades de Latinoamérica y algunas universidades alrededor del mundo se encontró que en la formación profesional predominan los cursos de introducción al mercadeo, mercadeo de los servicios, mercadeo estratégico, plan de mercadeo, mercadeo social, mercadeo en Internet, mercadeo ecológico, mercadeo internacional, psicología del consumidor, producto, promoción, precio e investigación de mercados. 
En el componente administrativo están la introducción a la administración, relaciones públicas, recursos humanos, gerencia empresarial, creación de empresas, derecho comercial y aspectos legales administrativos. La formación básica está basada principalmente en matemáticas, cálculo y estadística. En otras asignaturas se destacan la ética y la creatividad.

En lo relativo a los enfoques teóricos y las metodologías para el desarrollo curricular, se encuentran cambios importantes para aplicar a futuro en la malla curricular. Es innegable que la aproximación de las competencias, en sus diversas expresiones, tiene el papel protagónico y preside las principales reformas curriculares, así como la generación de importantes proyectos de investigación sobre procesos curriculares, de enseñanza, desarrollo y formación docente.

\section{Conclusiones}

El Ministerio de Educación Nacional se ha planteado un compromiso en el mejoramiento de la calidad de la educación superior, para lo cual propuso a las entidades educativas que incluyeran en sus proyectos la tarea de mejorar la calidad de sus programas y acceder a una certificación y acreditación de calidad.

El Ministerio de Educación Nacional formulo las competencias genéricas, las cuales deben ser transversales a todos los programas de formación en educación superior. Las competencias genéricas derivan en otras mas especializadas que finalmente lo que hacen es que contribuyen a la pertinencia social del educando en el mundo real empresarial.

Las competencias genéricas, deben complementarse con las competencias específicas que se requieren en los distintos programas de educación superior y su desarrollo debe ser integrador. Se entiende que la educación por competencias es una propuesta en la cual el punto de partida esta en identificar las competencias idóneas que debe desarrollar un estudiante y apropiarse de ellas al finalizar la carrera.
La propuesta de las competencias específicas es congruente y consistente con la propuesta educativa del gobierno basadas en pedagogías por competencias que tengan principios, fines y valores. Para ello se fortalecen los aspectos prioritarios importantes del compromiso de formar cada estudiante con procesos de reflexión, acción y solución a problemas que sean pertinentes con su carrera y de excelente desempeño en las organizaciones.

Por otra parte, para definir las competencias se consideraron los lineamientos curriculares de direccionamiento estratégico formulado por la Universidad investigada que está plasmado en su Misión, la cual consiste en integrar, con perspectiva internacional, las funciones sustantivas de docencia, investigación y proyección social para contribuir a la formación de personas con visión humanística, creativas y emprendedoras, a la generación de conocimiento y a la solución de problemas del entorno regional, nacional e internacional. También tiene como Visión, ser una Universidad consolidada, con desarrollos organizacionales propios de una institución reconocida regional y nacionalmente y cuya proyección internacional garantice la excelencia en sus programas de formación, la calidad y pertinencia de su investigación y proyección social y un alto nivel de integración de sus egresados con la sociedad en que viven y laboran.

En segundo lugar, se plantea la complejidad y laboriosidad que exige este proceso mediante el análisis comparativo de currículos, con tendencias nacionales e internacionales, lo que permitió constatar los elementos diferenciadores que cada plan curricular posee respecto de los demás. Desde un punto de vista estratégico, se seleccionaron los elementos que encajan en la categoría de fortalezas, en donde se observó claramente que las tendencias en el componente de formación del profesional de Mercadeo son: Plan de mercadeo, mercadeo de los servicios, mercadeo estratégico, mercadeo social, mercadeo en Internet, mercadeo ecológico, mercadeo internacional, psicología del consumidor, producto, promoción, precio e investigación de mercados. En el 
componente administrativo están las tendencias a la introducción a la administración, relaciones públicas, recursos humanos, gerencia empresarial, creación de empresas, derecho comercial y aspectos legales administrativos.

Para conocer las competencias se hicieron reuniones con estudiantes y los criterios predominantes se evidenciaron en la necesidad de tener asignaturas que interpreten la información cualitativa y cuantitativa para la toma de decisiones, identificar oportunidades y desarrollo de marcas en el mercado, sistemas de información para proyectos, negociar en escenarios nacionales e internacionales, utilizar tecnologías de información. Los egresados expresaron la necesidad de trabajar en casos de empresas reales PYMES, elaborar informes gerenciales, enfoques comerciales y énfasis en finanzas y costos. Los docentes determinaron que los elementos diferenciadores pueden estar dados en gestionar y evaluar estrategias de marketing, presupuestos, investigaciones de mercado y mercadeo electrónico.

El estudio de otros programas de formación en Colombia permitió evidenciar que otros componentes importantes son el pensamiento crítico, la comunicación oral y escrita, el emprendimiento, el trabajo bajo presión y en equipo, el liderazgo y unas excelentes relaciones interpersonales. Con base en lo anterior, el diseño curricular desde el enfoque complejo para el programa de Mercadeo y Negocios Internacionales pretende formar profesionales integrales, con un claro proyecto de vida, con un espíritu creativo, investigativo, capaz de formular y desarrollar estrategias dentro de un entorno complejo, competitivo y retador. Se trata de formar un profesional con las competencias adecuadas para desempeñarse con idoneidad en diversos campos de su quehacer profesional.

\section{Referencias}

Comisión Europea (2004). Competencias clave para un aprendizaje a lo largo de la vida. Un marco de referencia europeo. Bruselas. Dirección General de Educación y Cultura (Grupo de Trabajo B "Competencias Clave"). Disponible en: http:// europa.eu/scadplus/leg/es/cha/c11090.htm

Chyung, S.Y., Stepich, D. \& Cox. D. (2006). Building a CompetencyBased Currículo Architectura to Educate 21st-Century Business Practicioners. En: Journal of Education for Business, (6) 81: 307-311.

Corominas, E. (2001). Competencias genéricas en la formación universitaria. Revista de Educación (325), 299-321.

Echeverria, B., Isus, S. \& Sarasola, L. 2001. Formación para el desarrollo de la profesionalidad. Tesalónica, CEDEFOR.

Gonzalez, J. \& Wagenaar, R. (2003): Tuning Educational Structures in Europe. Informe Final - Proyecto Piloto, Fase 1, Bilbao, Universidad de Deusto.

Le Bofert, G. (1994). De la competence. Essai sur unattracteur étrange. Paris Les Editions d'organization.

Morín, E. (1994). Introducción al pensamiento complejo. Barcelona: Gedisa, p. 118.

Morín, E. (1996) "En el pensamiento complejo contra el pensamiento único", entrevista realizada por Nelson Vallejo Gómez, en Sociología y Política, Nueva época, año IV, núm. 8, México.

Morin, E. (1999). Los siete sobres necesarias a la educación del futuro. Paris: UNESCO.

OECD (2003) Education and the economy in a changing society. Paris: OECD.

Rey, B. (1996). Les compétences transversales en question. Paris: ESF éditeurs.

Schiro, M. (1978). Curriculum for better schools. the great, ideological debate. Engle Wood Cliffs. Educational technology publication.

Sladogna, M. (2000). Una mirada a la construcción de las competencias desde el sistema educativo. La experiencia Argentina. Boletín técnico. CINTERFOR-OIT. Competencias laborales en la formación profesional. Interamericano de Formación Profesional, $\mathrm{N}^{\circ} 149$.

Stenhouse, L. (1988) Investigación y desarrollo del curriculum. Madrid: Morata.

Tobón, S. (2010). Formación integral y competencias. Pensamiento complejo, currículo, didáctica y evaluación. Bogotá: ECOE.

Universidad Católica de Colombia (2005). Coordinación ciencias básicas. Disponible en: http://portalweb.ucatolica.edu.co/ easyWeb2/informes/documentos/ACTA\%20\%20\%20022.\%20 \%2026-09-05\%20CB\%20ET.pdf 
